\title{
Acquired cupping of the optic nerve head in normotensive eyes
}

\author{
J. THEODORE SCHWARTZ, $\dagger$ FRANK H. REULING $\dagger$, AND ROBERT J. GARRISON* \\ From the National Eye Institute $\dagger$ and the National Heart and Lung Institute*, \\ The National Institutes of Health, Bethesda, Maryland 20014, U.S.A.
}

This study of a horizontal cup/disc ratio (GDR-H) among a sample of normal subjects calls attention to several likely sources of acquired enlargement and moulding of the physiological cup in normotensive eyes. Some of the findings are new, but the chief purpose of this report is to emphasize the likelihood that the physiological cup of the "normal" eye is not a static structure and that greater understanding of acquired changes in this least studied affiliate of the glaucoma triad is needed.

\section{Methods}

Glinical measurements of a CDR-H were obtained as part of a base-line examination of 160 twin subjects who participated in a study on the ocular hypertensive response to topical dexamethasone, described previously (Schwartz, Reuling, Feinleib, Garrison, and Collie, 1972; r973a, b). Methods of identifying the twins, selecting the sample, and performing tonometric measurements have been described in earlier reports.

In this study, CDR-H was taken as the ratio of the widest horizontal diameter of the optic cup to the widest horizontal diameter of the optic disc, estimated to the nearest tenth. This designation was adapted from that described by Snydacker (1964) and modified by Armaly (1967). The demarcation from papillary surface to optic

Address for reprints: J. Theodore Schwartz, M.D., Section on Ophthalmic Field and Development Research, Office of Biometry and Epidemiology, National Eye Institute, The National Institutes of Health, Bethesda, Maryland 20014, U.S.A. cup was distinguished as the location of that imaginary contour line which best represented the first discernible change in surface curvature as the surface of the optic nerve head coursed posteriorly at the rim of the cup. The range in shapes of physiological cups observed in this study sample formed several groups, two of which merit special mention.

(r) If a definite central depression was present, but curvature of the temporal surface of the nerve head increased gradually from the optic rim to the floor of the depression without an identifiable demarcation within the broad, gently sloping curve, then the optic rim was taken as the temporal margin.

(2) For eccentric or irregular cups, the widest horizontal cup and disc diameters were used whether they both occurred along the same straight line or not.

To measure CDR-H, pupils were dilated using three instillations of I per cent. Mydriacyl (Alcon Laboratories) at 5-min. intervals. All measurements were made clinically by one examiner (FHR), and were based on a stereoscopic view of the optic nerve head using the AllenThorpe contact lens with the Haag-Streit biomicroscope.

Refractions described in this report were obtained by ordinary clinical procedures without the use of a standardized protocol. All but 23 of the 160 refractions were obtained under cycloplegia. All exceptions were either myopic or of age $\geqslant 45$ years.

\section{Results}

The age, sex, and race of the sample are given in Table I. The frequency distribution of CDR-H for

Table I Study sample of 80 twin pairs characterized by age, sex, and race

\begin{tabular}{|c|c|c|c|c|}
\hline \multirow{2}{*}{$\begin{array}{l}\text { Age } \\
\text { (yrs) }\end{array}$} & \multicolumn{2}{|l|}{ Sex } & \multicolumn{2}{|c|}{ Total pairs } \\
\hline & Male & Female & No. & Per cent. \\
\hline I $5^{-24}$ & $26(2)$ & $27(3)$ & 53 & $66 \cdot 25$ \\
\hline $25-34$ & $2(\mathrm{I})$ & $5(1)$ & 7 & $8 \cdot 75$ \\
\hline $35-44$ & 4 & $3(1)$ & 7 & $8 \cdot 75$ \\
\hline $45+$ & 3 & Io (I) & I3 & $16 \cdot 25$ \\
\hline Mean $25 \cdot 6$ & 35 & 45 & $80(9)$ & 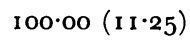 \\
\hline
\end{tabular}

( ) Represents black pairs contained in group 
all 320 normal eyes included in this investigation is shown in Fig. I. The extent to which the distribution of CDR-H conforms to a normal or Gaussian configuration can be assessed from Fig. 2, where the same data are replotted as a percentage cumulative frequency distribution on a probability ordinate scale. By this method, a Gaussian configuration will appear as a straight line. The data do not deviate materially from a Gaussian distribution.

Differences in CDR-H, as observed between the right and left eyes of these subjects, are summarized in Table II.

As shown in Table III, the average CDR-H was very similar when compared by laterality, sex, and race. However, relatively few non-white subjects were studied.

The association between CDR-H and age, intraocular pressure (IOP), and refractive error was studied by regression analysis. Results are summarized in Table IV. Since in the present study the subjects

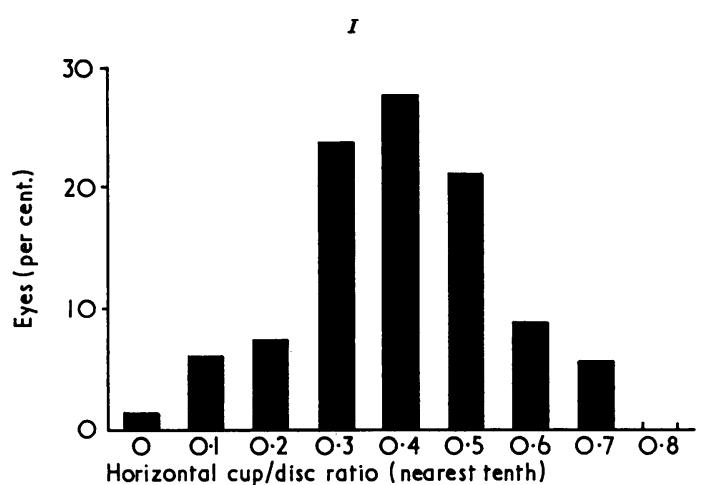

Table II Distribution of paired differences between horizontal cup/disc ratio of right eye and left eye for 160 subjects

\begin{tabular}{|c|c|c|}
\hline $\begin{array}{l}C D R-H(\text { Right }) \\
\text { minus } \\
C D R-H(\text { Left })\end{array}$ & $\begin{array}{l}\text { No. of } \\
\text { subjects }\end{array}$ & $\begin{array}{l}\text { Percentage } \\
\text { of } \\
\text { total }\end{array}$ \\
\hline-0.2 & 7 & $4 \cdot 4$ \\
\hline$-0 \cdot I$ & I 8 & II 3 \\
\hline $0 \cdot 0$ & I I 4 & $71 \cdot 2$ \\
\hline+0.1 & 17 & $10 \cdot 6$ \\
\hline+0.2 & 4 & $2 \cdot 5$ \\
\hline
\end{tabular}

2

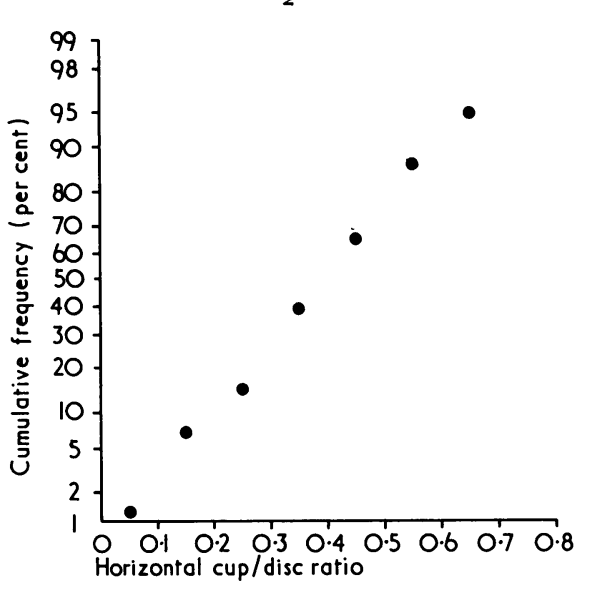

FIG. I Percentage frequency distribution of horizontal cup/disc ratio for 320 eyes

FIG. 2 Data from Fig. I shown as percentage cumulative frequency distribution using a probability ordinate scale.

In this cumulative distribution, abscissa values are plotted at the end of corresponding intervals on the original histogram, i.e. at values mid-way between successive bars of Fig. I

Table III Means and standard deviations of horizontal cup/disc ratio by laterality, race and sex

\begin{tabular}{|c|c|c|c|c|}
\hline \multirow{2}{*}{\multicolumn{2}{|c|}{ Variable }} & \multirow{3}{*}{$\begin{array}{l}\text { No. of observations } \\
80 \\
80\end{array}$} & \multicolumn{2}{|l|}{$C D R-H$} \\
\hline & & & Mean value & Standard deviation*** \\
\hline Laterality* & $\begin{array}{l}\text { Right } \\
\text { Left }\end{array}$ & & $\begin{array}{l}0 \cdot 40 \\
0 \cdot 39\end{array}$ & $\begin{array}{l}0 \cdot 133 \\
0 \cdot 137\end{array}$ \\
\hline Race** & $\begin{array}{l}\text { White } \\
\text { Non-white }\end{array}$ & $\begin{array}{r}71 \\
9\end{array}$ & $\begin{array}{l}0 \cdot 39 \\
0 \cdot 38\end{array}$ & $\begin{array}{l}0 \cdot 127 \\
0 \cdot 178\end{array}$ \\
\hline Sex** & $\begin{array}{l}\text { Male } \\
\text { Female }\end{array}$ & $\begin{array}{l}35 \\
45\end{array}$ & $\begin{array}{l}0.40 \\
0.39\end{array}$ & $\begin{array}{l}0 \cdot 146 \\
0 \cdot 123\end{array}$ \\
\hline
\end{tabular}

\footnotetext{
* Average for both right or both left eyes of the pair

** Average of four eyes for the pair

*** Standard deviations are based on mean value of CDR-H for two or four eyes (as indicated) and are therefore smaller than expected for the same number of simple independent measurements.
} 
Table IV Statistical summary of tests on horizontal cup/disc ratio $(C D R-H)$, age, intraocular pressure (IOP), and spheric equivalent refraction (REF) among 80 pairs of twins

\begin{tabular}{|c|c|c|c|c|}
\hline \multirow[b]{2}{*}{ Variable } & \multirow{2}{*}{$\begin{array}{l}\text { Correlation } \\
\text { coefficient }\end{array}$} & \multicolumn{3}{|c|}{ Regression coefficient } \\
\hline & & Value & $\mathbf{t}$ & $P$ \\
\hline GDR-H/Age & 0.337 & $+0.0035 \times$ Age & 3.17 & $<0.01$ \\
\hline CDR-H/IOP & $0 \cdot 322$ & $+0.018 \times 1 O P$ & $3 \cdot 00$ & $<0.01$ \\
\hline CDR-H/REF & -0.169 & $-0.012 \times \mathrm{REF}$ & $-1 \cdot 51$ & $0.1<P<0.2$ \\
\hline IOP/Age & $0 \cdot 346$ & $+0.064 \times$ Age & $3 \cdot 26$ & $<0.01$ \\
\hline \multirow[t]{2}{*}{ CDR-H/Age, IOP } & 0.402 & $+0.0026 \times$ Age & $2 \cdot 31$ & $<0.05$ \\
\hline & & $+0.013 \times 1 O P$ & $2 \cdot 10$ & $<0.05$ \\
\hline
\end{tabular}

Distribution of individual variables among eighty pairs

\begin{tabular}{lcc}
\hline Variable & \multicolumn{1}{c}{ Mean } & Standard deviation* \\
\cline { 2 - 3 } CDR-H & 0.39 & $0 \cdot 13$ \\
IOP & 13.79 & $2 \cdot 38$ \\
REF & -0.52 & $1 \cdot 84$
\end{tabular}

* Based on means of four eyes for each of eighty pairs and therefore smaller than expected for same number of single independent readings

were twin pairs rather than random non-siblings, all regressions in Table IV were calculated on an average value for each of the eighty pairs, thereby regarding each twin pair as a single value. This method effectively removes a potential source of error in the regression calculations which could result from significant intrapair correlations among twins. On the assumption that members of twin pairs are not different from individuals in their relationship to the variables under study, estimates of regression coefficients obtained from the averaged data should not differ materially from estimates obtained among individuals. A significant regression was found for CDR-H on age and IOP but not on refractive error. Scatter diagrams representing these data are given in Figs 3, 4, and 5. The regression of IOP on age, shown in Table IV, shows that these two variables are themselves associated. Accordingly, the partial effects of age and IOP in relation to CDR-H were examined by a multiple regression. As shown in Table IV, the multiple regression of CDR-H on age and IOP was also significant. A step-down multiple regression with refraction as an additional independent variable demonstrated that refraction did not contribute significantly to the prediction of CDR-H for this sample. As shown in Fig. 6, however, few of these examinees exhibited a large refractive error.

3
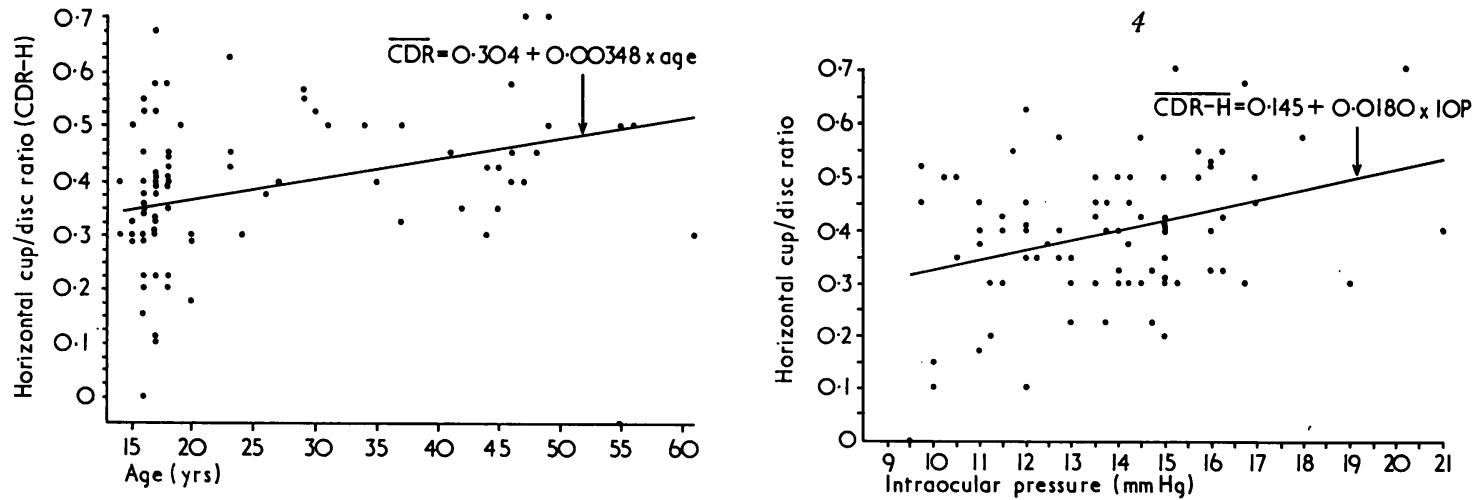

FIG. 3 Horizontal cup/disc ratio v. age for eighty twin pairs. Average values for the pair are based on individual averages for right and left eyes

FIG. 4 Horizontal cup/disc ratio v. intraocular pressure for eighty twin pairs. Average values for the pair are based on individual averages for right and left eyes 

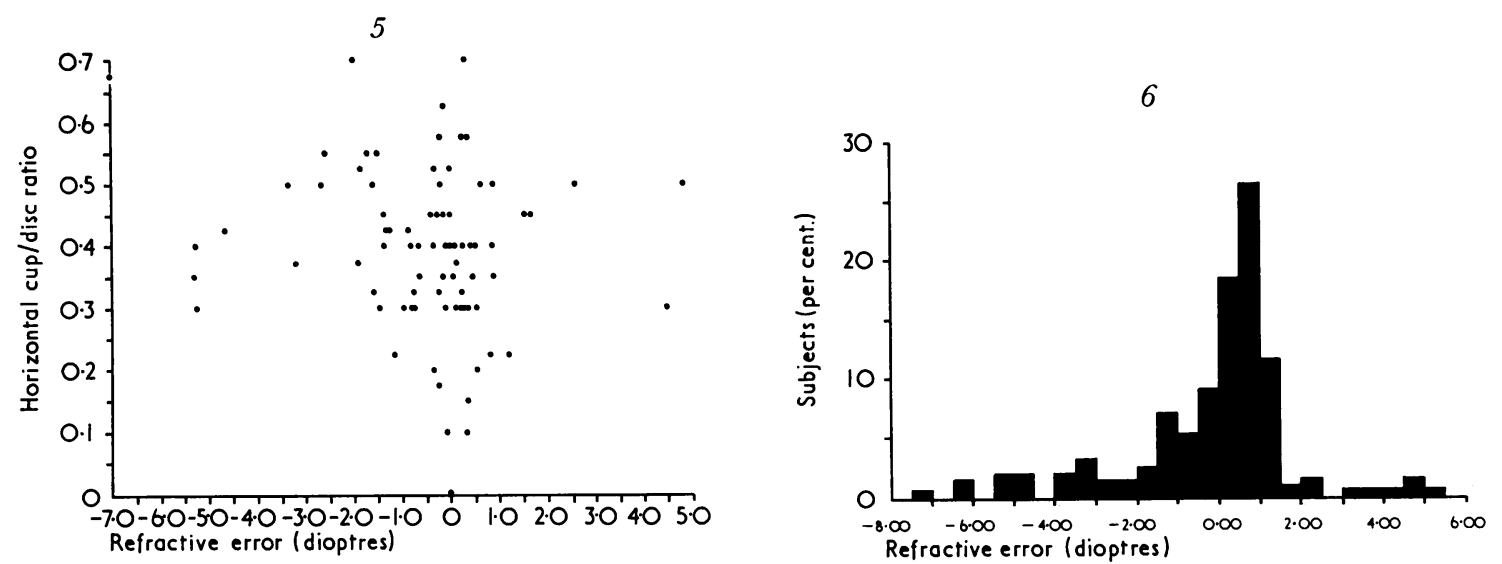

FIG. 5 Horizontal cup/disc ratio v. refractive error for eighty twin pairs. Average values for the pair are based on individual averages of spheric equivalent for right and left eyes

FIG. 6 Frequency distribution of refractive error among I6o twin subjects. Each subject represented by average spheric equivalent for both eyes

\section{Discussion}

Three findings in this study are of clinical interest with respect to acquired cupping in normotensive eyes:

(I) The significant association between CDR-H and age when the effect of pressure is partialled-out;

(2) The significant association between CDR-H and pressure when the effective of age is partialledout;

(3) The absence of a significant association between GDR-H and small to mid-range values of ametropia.

\section{Age v. cup size}

The relative size of the physiological cup in comparison to the optic nerve was first expressed as a ratio by Pickard (192I), whose original report stands as an early example of careful clinical and study design methods. Using an estimate of cup/disc ratio based on relative areas (CDR-A), Pickard reported that the physiological cup in normal subjects enlarged with age. His active interest in this question persisted for many years (Pickard, 1923, 1931, 1935), as did his belief in a significant age effect. His final report (Pickard, 1948) included data on a cohort of 234 eyes followed prospectively for 15 years and also an estimate of the average rate of increase in CDR-A with age.

Later investigators reported mixed findings with respect to an age effect. Using different methods of estimating cup size, Colenbrander (1960), Ford and Sarwar (1963), and B. Schwartz, Reinstein, and Lieberman (1973) observed a significant increase in cup size with age, whereas Snydacker (1964), Hollows and McGuinness (1967), and Armaly
(1967) did not. Armaly and Sayegh (1969) subsequently noted larger horizontal cup/disc ratios among older subjects, but concluded that the finding reflected a higher intraocular pressure in the older age group. It is popularly taught (Kolker and Hetherington, 1970) at the present time that, contrary to earlier reports in the literature, cupping does not increase with age. The finding of a significant association between CDR-H and age when considered apart from an effect of intraocular pressure has not been reported in the past.

Table IV shows that the simple regression coefficient of CDR-H on age is 0.0035 CDR-H per year for this particular sample of subjects, whereas the partial regression coefficient is 0.0026 . It is noteworthy that Pickard ( 1948 ) originally reported an increase of CDR-A for his sample in the amount of o.35 percentage CDR-A units (pressure effect not partialled). In order to compare our data with those of Pickard, we converted our full set of CDR-H measurements to values of percentage CDR-A units (assuming a circular disc and cup), and the findings of both studies were comparable. Annual increase in percentage area ratio, as estimated from our data, became 0.27 per cent. as compared with Pickard's observed 0.35 per cent.

The partial regression coefficients for age and IOP in the multiple regression equation provide a quantitative estimate of the average effect of each of these variables apart from the other. Thus, partial regression coefficients represent relatively "pure" effects. In an applied perspective, a difference in age between 15 and 60 years, as observed in this sample, would be equivalent to average differences in CDR-H of 0.16 and 0.12 , based on the simple and partial regression coefficients respectively. (Such prospective consideration of "cross-sectional" 
data assumes that the natural history of the phenomenon is stable in the population and is independent of the year of birth.)

Coefficients of the regression equations reflect an average association between CDR-H and age for the sample. The clinician, however, is not concerned with statistical averages when he attempts to diagnose his patients. He needs to know something about the higher ranges of age effect as normally expected among individual subjects. Several additional considerations are involved. "Average" effects would, of course, mis-state by varying amounts the association between age and cup size for many individuals comprising a given sample. Secondly, the average effects observed in Table IV pertain to this particular sample. Other samples might show higher or lower average change in CDR-H with age. Thirdly, although CDR-H is regarded as increasing linearly with age in the regression equations, the present data cannot be regarded as sufficient to establish linearity over the range of ages studied, nor do they provide information regarding individuals under 15 or over $6 \mathrm{I}$ years of age. Accordingly, the possibility of contrasting age effects for portions of the complete age spectrum cannot be ruled out by these data. From these statistical perspectives, it is reasonable to anticipate that some normal individuals are likely to exhibit an increase in CDR-H with age which would be materially greater than expressed by the average cited in Table IV. It might also be expected, simply on the basis of biological variation, that the optic nerve heads of different individuals would be subject to varying degrees of stress and varying degrees of resistance to tissue loss in association with ageing, whatever the underlying pathophysiological mechanism. A highly relevant statistical observation was again contributed by Pickard (1948), who published a description of nine types of increase in CDR-A which he observed among individuals during their 15-year follow-up. Pickard did not establish that his nine example cases comprised a representative sample of all 234 eyes studied, but four of his nine examples showed rates of increase in CDR-H (converted from GDR-A) of as much as 0.02 to 0.05 per year. This is equivalent to a change in CDR-H for some "normal" individuals in the amount of two full tenth units over a period as short as 4 years. Thus, on the basis of available information as well as on theoretical grounds, it appears that a possible "normal" enlargement of the physiological cup with advancing years should be given consideration when the clinician uses change in cup size as an element in his diagnosis of glaucoma.

\section{Intraocular pressure v. cup size}

Few investigators have examined the relationship between cup size and intraocular pressure among healthy "normotensive" eyes. In their study at Rhondda Fach, Hollows and Graham (I966a, b) observed that the optic cups in subjects with ocular hypertension without field loss were not distinguishable from those of normotensive individuals. In a further analysis of the specific relationship between cup size and prevailing ocular tension among normotensive subjects, Hollows and McGuinness (1967) observed no significant association. In a later study among normotensive subjects, Armaly and Sayegh (1969) reported an increase in horizontal cup/disc ratio with higher levels of applanation pressure which was significant when tested by nonparametric methods. They reported no age effect. The finding among normal subjects of a significant association between CDR-H and intraocular pressure with age effect partialled-out, and a quantitative estimate of the pressure effect have not been reported in the past.

The regression equations in Table IV pertaining to pressure can be interpreted in similar fashion to those for age. The simple regression coefficient of CDR-H on pressure is 0.018 , whereas the partial regression coefficient for pressure with age partialledout is 0.013. A general "feel" for the relative "potency" of the variables age and pressure can be obtained using the information in Table IV. From the regression of IOP on age, it can be estimated that average pressure levels corresponding to the age range 15 to 60 years for this sample would be approximately I $3 \cdot \mathrm{I}$ to $\mathrm{I} 6 \cdot 0 \mathrm{~mm}$. $\mathrm{Hg}$. The slope of the partial regression of GDR-H on IOP, suggests that this difference in pressure is equivalent to an average difference in GDR-H of approximately 0.04. The average difference in CDR-H associated with the partialled age effect across this same time base was previously estimated at $0 \cdot 12$. This comparison further supports the concept of a relatively important age effect among normal eyes.

Earlier comments with respect to age effect also apply to the variable pressure. Some normal individuals would be expected to be more susceptible to change than the average. These observations lend support to the need for a clinical awareness of potential change in contour of the "normal" physiological cup and for a better understanding of such change.

\section{Refractive error v. cup size}

It is of interest that community-based studies and studies among randomly selected ophthalmic patients without ocular disease have commonly failed to demonstrate a strong association between refractive status and horizontal cup diameter. This is true of studies by Hollows and McGuinness (1967) and by Snydacker (1964) as well as our own. In their series of routine examinees without pathological discs, Ford and Sarwar (1963) classified physiological 
cups as absent or small more commonly in hyperopic eyes and as large more commonly in myopia with astigmatism. A full quantitative description of this difference was not given, however, and these investigators did not include refractive error in their concluding description of variables associated with cup size. Tomlinson and Phillips ( 1969 ), in a careful comparison of cup morphology with axial length and refractive error among a sample of students, found a negative association between area CDR and refractive error which was significant at the $\mathrm{P}<0.05$ level. The association they observed was not statistically significant when the same subjects were compared on the basis of horizontal CDR and refractive error.

Flattening of the temporal side of the optic nerve head is regarded as a characteristic of myopia (Hogan and Zimmerman, 1962), and it is commonly held on the basis of clinical experience that the more (axially) myopic an eye, the larger the cup in relation to the whole disc (Tomlinson and Phillips, 1969). The common failure to detect an obvious statistical association between horizontal cup diameter and refractive error among samples of normal subjects may be partly explained by the relatively low proportions of highly ametropic individuals contained in such samples. In any event, current evidence does not appear to support a strong association between horizontal cup diameter and ametropia up to midrange values. If the physiological cup is materially wider in highly myopic eyes, as commonly held, then the findings of this and foregoing studies would be consistent with an acquired widening occurring relatively late in the course of myopia, i.e. not until the condition progresses to higher levels.

It is recognized clinically that myopic discs are difficult to interpret with respect to glaucoma (Chandler and Grant, I965) and the wide cup and/or "myopic" nerve head is regarded as unusually sensitive to progressive field loss in glaucoma (Kolker and Hetherington, 1970; Levene, I 97 I). A likelihood of acquired change in the contours of highly myopic cups in otherwise normal eyes, however, is not generally emphasized, and much potentially helpful natural history of change at the optic nerve head in ametropia remains unknown. For example, at what level(s) of myopia may the clinician begin to anticipate acquired changes at the nerve head? Are myopic changes strictly confined to the possibly separate "malignant" entity? What is the quantitative spectrum of change in cup-size among normotensive subjects, given the combined variables of age and high refractive error? Does myopic change at the optic nerve head necessarily terminate when and if the myopic refraction stabilizes, i.e. may the myopic nerve head itself be more susceptible to changes associated with age and the "normal" range of IOP? Comparative data of this type are needed to assess, in turn, the concept of increased susceptibility of the myopic disc to deformity from glaucoma.

\section{General comment}

That the physiological cup in normotensive subjects is not to be regarded as a fixed sculpture merits emphasis on the basis of the preceding analysis. It appears that at least three variables (age, prevailing "normal" pressure, and, by inference, high myopia) are in themselves and in combination associated with an acquired moulding or widening of the physiological cup in otherwise healthy eyes. It is unwise to ignore the degree of acquired change in some normal eyes in the clinical diagnosis of glaucoma. The possibility of "normal" cupping does not suggest that the clinician should lightly disregard an acquired disc change; rather, it calls attention to the importance of an adequate central visual field examination in diagnosing glaucoma. Greater understanding of the limits of "normal" cupping is now seen as a prerequisite to the resolution of long-standing clinical questions-for example, whether "morbid" cupping precedes or follows the visual field defects of glaucoma. Present data respecting "normal" cupping are sparse. This seems to be due to the difficulty encountered in documenting the configuration of the physiological cup in a manner which is sufficiently accurate, valid, and reliable (Schwartz, in press) and which lends itself to available methods of data analysis. The possibility of developing appropriate studies to assess changes at the nerve head in normal eyes, in ametropia, and in glaucoma itself, would be enhanced by improved clinical and instrumental methods of documenting the configuration of the optic nerve head in a computercompatible format. With increasing use of fundus photography in clinical practice (Kolker and Hetherington, 1970), it will become more imperative that "normal" and "morbid" moulding of the optic nerve head be better defined and differentiated.

\section{Summary}

Clinical measurements of a horizontal cup/disc ratio were obtained among a sample of 160 normal twin subjects by stereoscopic biomicroscopy. Measurements of horizontal cup/disc ratio were normally distributed and there was no significant association with sex, laterality, race, or refractive error in the mid-range. However, there was a significant association between size of the cup/disc ratio and age as well as intraocular pressure. After taking into account the correlation between age and intraocular pressure in normal eyes, the association between cup/disc ratio and each of these variables remained statistically significant. It is not always recognized that the size of the normal physiological cup increases with age, and 
it was not previously observed that "normal" cupping occurs with age apart from the effect of pressure. The average effect of age on cup/disc ratio, amounting to an increase of 0.0026 per year for this sample, is small. But, allowing for individual variation, some persons could show a recognizable acquired increase in cup/disc ratio with age, even in the absence of glaucoma. The possibility of "normal" cupping occurring in association with age, pressure, or high myopia, alone or in combination, merits consideration in the clinical evaluation of glaucoma suspects and serves to re-emphasize the importance of an adequate central visual field examination in the diagnosis of glaucoma. Studies are needed on the differentiation between "normal" and "morbid" moulding of the optic nerve head.

\section{References}

ARMaly, M. F. (1967) Arch. Ophthal. (Chicago), 78, 35 and SAYEGH, R. E. (I969) Ibid., 82, I9I

GHANDLER, P. A., and GRANT, w. M. (1965) "Lectures on Glaucoma". Lea and Febiger, Philadelphia COLENBRANDER, M. C. (1960) Ophthalmologica (Basel), 139, 491

FORD, M., and SARWAR, M. (1963) Brit. F. Ophthal., 47, 50

hogan, M. J., and zimmerman, L. E. (1962) "Ophthalmic Pathology. An Atlas and Textbook", 2nd ed.

Saunders, Philadelphia

Hollows, F. C., and GRAHAM, P. A. (1966a) Brit. F. Ophthal., 50, 570 and - (1966b) "Glaucoma", ed. L. B. Hunt. Livingstone, Edinburgh and McGuinness, R. (1967) Trans. ophthal. Soc. Aust., 1966, 25, 38

KOLKeR, A. E., and HETHERINGTON, J. (revisers) (1970) "Becker and Shaffer's Diagnosis and Therapy of the Glaucomas", 3rd ed. Mosby, St. Louis

LEVENE, R. Z. (1971) Arch. Ophthal. (Chicago), 85, 227

PICKARD, R. (I92 I) Proc. roy. Soc. Med., 14 (Sect. Ophthal.), 3 I

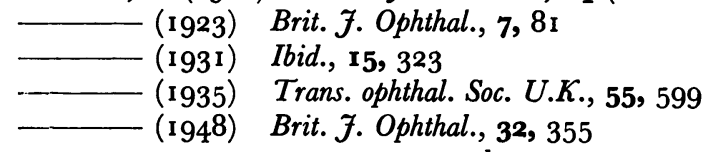

SGHWARTZ, B., REINSTEIN, N. M., and LIEBERMAN, D. M. (1973) Arch. Ophthal. (Chicago), 89, 278

SCHWARTZ, J. T. "The influence of methodologic differences on measurements of cup/disc ratio. An epidemiologic assessment". (Submitted for publication)

—, REUling, F. H., FEINLEIB, M., GARRISON, R. J., and collie, D. J. (1972) F. med. Genet., 9, I 37

SNYDACKer, D. (1964) Amer. F. Ophthal., 58, 958 ,

tomlinson, A., and PHILlips, c. I. (I969) Brit. 7. Ophthal., 53, 765 